\title{
A Force Feedback System for Steer-by-Wire Applications Based on Low-Cost MR Fluids - Design Hints
}

\author{
L.Peretti, M.Zigliotto \\ Dept. of Engineering and Management, University of Padova \\ Stradella S. Nicola, 3 - 36100 Vicenza, Italy \\ Email: mauro.zigliotto@unipd.it
}

Keywords: Steer-by-Wire, magnetorheological fluids, forcefeedback, electric drives for automotive.

\begin{abstract}
In the automotive research scenario, the main purpose of a steer-by-wire system is the elimination of all mechanical connections between wheels and the steering wheel. The driver gets just a virtual and configurable feedback through the steering wheel, which becomes a stand-alone component. Among the emerging alternatives, magnetorheological (MR) fluids exhibit interesting properties that could well fit for safe and reliable force feedback systems. Through the collected data, this paper offers the essential information for the realisation of a force feedback device for steer-by-wire application. The paper includes the design and test of a preindustrialised system, exploited for the measurements of lowcost, homemade MR fluids. Experimental results and their discussion conclude the paper.
\end{abstract}

\section{Introduction}

In the last decade, the share of electronic components in automotive has been in constant growth. Among the several attracting proposals, electric power steering (EPS) represents a substantial improvement with respect to conventional hydraulic power steering. EPS makes use of an electric motor to augment the mechanical forces created when the driver moves the steering wheel. The surviving link between tires and the steering wheel guarantees the appropriate force feedback. As a step beyond, steer-by-wire (SBW) approach $[2,3]$ aims to eliminate that link definitively. In comparison with conventional steering, SBW may reduce the annoying steering wheel vibrations, while the steering feel can be customised according to user's demand. Since it has been demonstrated that the human brain recognises tactile signals much faster than visual inputs, preserving tactile feedback in a SBW system is however essential for safety and driver acceptance. An open issue is how to reproduce the missing feedback link. A first choice is the use of a current-controlled electric motor coupled to the steering wheel, as depicted in Fig.1 [2]. The steering control system delivers the torque reference to the synchronous permanent magnet motor (SPM), aiming at reproducing the torque at the wheels. If only passive (in the sense of braking) torque are of interest, Rheonetic $^{\mathrm{TM}}$ Tactile Feedback Devices, manufactured by
Lord Co. (UK), represent a smart solution [9]. Based on magnetorheological (MR) fluids, they give high fidelity steering feel through continuously resistive, rather than active, force. In case of fault, a feedback electric motor could transmit to the driver unwanted and potentially hazardous feedback forces. It is evident how a MR-based force feedback system can substitute the pair inverter-SPM motor of Fig.1, with inherent advantages in terms of reliability and safety.

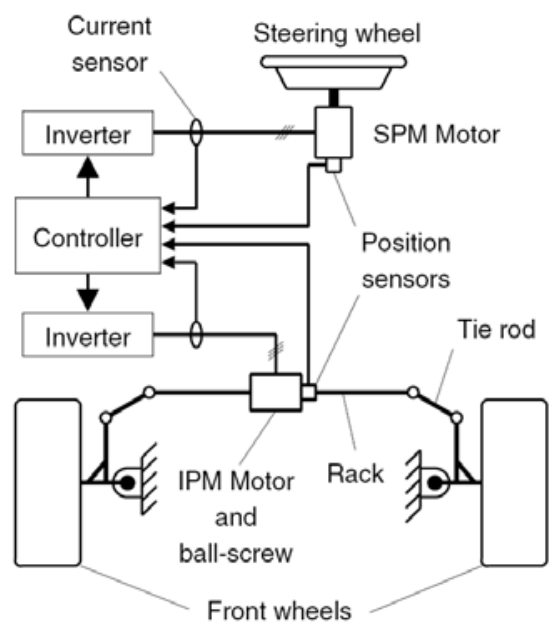

Fig. 1. Scheme of a steer-by-wire system with active force feedback.

Even if the technical literature reports examples and instructions for preparing homemade MR-fluids [4], to the authors' knowledge there is a lack of information on how these fluids can be tested and characterised. An example, on a side topic, is reported in [6], in which the elastic modulus of solid MR composites is measured with a torsion test under various magnetic field strengths. With the aim of covering the gap, this paper reports the detailed analysis and design of a pre-industrialised prototype, for the characterisation of homemade, low-cost MR fluids. Through the collected data, the proposed system also gives essential information toward the production of an engineered force feedback device for steer-by-wire application. The paper includes a complete set of experimental results.

\section{Basic concepts of MR fluids}

Controllable fluids can be divided into two major categories: electrorheological (ER) and magnetorheological fluids. The former change their rheological properties under an electric 
field application, while the latter are sensitive to magnetic fields. Both of them are suspensions of polarizable iron particles having a size of few microns. However, ER fluids are less used because of their limitation in term of stability, temperature range, energy density and voltage requirements, especially for automotive applications (2-5 kV for ER fluids against 2-25 V for MR fluids, with a power requirement of 2$50 \mathrm{~W}$ for both) $[5,7]$.

\subsection{Recalls of rheology and fluidodynamics}

Rheology studies material properties like elasticity, viscosity and plasticity. When dealing with controllable fluids, the attention is focused on viscosity. First, it is essential to define the shear stress $T$ as the force per unit area required to sustain a constant rate of fluid movement, and the shear rate $\dot{\gamma}$ as the spatial gradient of velocity of the fluid, that is the rate of change of velocity at which one layer of fluid passes over an adjacent layer along. A common relation between shear rate and shear stress has been formulated by I.Newton (16421727), and describes the behaviour of Newtonian fluids like water, air or other oils:

$$
T=\eta \dot{\gamma}
$$

where $\eta$ is the dynamic viscosity. If a shear stress $T$ is applied onto the superficial fluid layer, the latter moves itself with a velocity $u(y)$ and a spatial gradient $d u / d y=\dot{\gamma}$, inversely proportional to the fluid's viscosity $\eta$. Internal fluid layers move themselves with decreasing velocity until the last and standstill layer, which is in direct contact with the steady plate. But Newton's viscosity law is not completely adequate for the description of MR fluids behaviour. The required extension is represented by the Bingham-Green model [7], which describes the behaviour of viscoplastic fluids that have intermediate characteristics between solid plastic materials and Newtonian fluids. In the Bingham-Green model, the relationship between $T$ and $\eta$ is expressed by

$$
T=T_{c}(H) \operatorname{sgn}(\dot{\gamma})+\eta \dot{\gamma}
$$

where $T_{c}$ is the magnetic-dependent yield stress. It behaves similarly to a Coulomb friction, in the sense that the shear stress required for a little movement of the uppermost layer of Fig.2 must exceed $T_{c}$. Above $T_{c}$, the fluid behaves like a Newtonian fluid, since $T_{c}$ has no influence on $\eta$.

\subsection{MR fluids properties}

The data on both magnetic and rheological properties of MR fluids are essential for a proper design of any MR fluid-based device. Magnetic B-H curves of most MR fluids are approximately linear up to an applied field of about $0,02 / \mu_{0}$ $\mathrm{A} / \mathrm{m}$, where $\mu_{0}$ is the vacuum permeability and little or no hysteresis can be observed [8]. From a rheological point of view, as no external magnetic field is applied, iron particles within the MR fluid arrange themselves in a random manner, as shown in Fig.2. In a MR-fluid, the application of an external magnetic field polarizes the iron particles suspended in a non-conductive liquid. The interaction between the resulting dipoles causes the particles to form columnar structures parallel to the applied field.

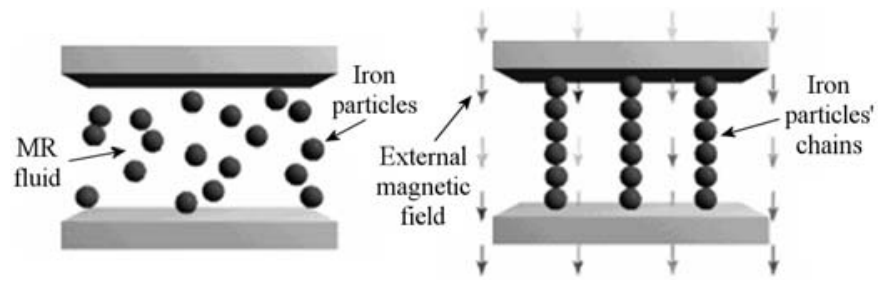

Fig. 2. MR fluids behaviour.

These chain-like structures restrict the fluid's motion, offering an high passive resistance to any external horizontal force and thereby increasing the viscous characteristics of the suspension [7]. In the absence of an applied field, the fluids exhibit Netwonian-like behaviour. Yield stress' range of commercial MR fluids can be quite high (30 to $100 \mathrm{kPa}$, [5]), and its contribute to the total shear stress is preponderant with respect to $\eta \dot{\gamma}$, which is sensitive to temperature variations, but its value is normally limited to few tens of Pa only.

\section{The proposed MR fluid}

As mentioned, a MR fluid essentially consists of magnetically polarizable particles, a carrier liquid, and a stabilising agent that helps to inhibit particles' settlement [4]. It is not difficult to prepare a homemade MR fluid using common ingredients, although there are some constraints to keep in mind. Particles should have the highest magnetic saturation for a strong MR fluid, and their size is fundamental, because too large particles will rapidly settle out of suspension, while too small ones will weaken the MR effect. The best available particles are alloys of iron and cobalt that have saturation magnetization of about $2.4 \mathrm{~T}$. Unfortunately, such alloys are prohibitively expensive for most practical applications. High purity elemental iron in the range of 1 to $10 \mu \mathrm{m}$ is suitable, but always for cost issues it is better to fall back on simpler solutions using $100 \mu \mathrm{m}$ (130 mesh) to $300 \mu \mathrm{m}$ (50 mesh) iron particles. These filings are actually larger than ideal, but they are quite easily obtainable and it has been found that often the MR performance remains acceptable. The main drawback is a reduced sedimentation stability, which compels to shake the MR fluid frequently. Two photos of the iron filings used in the following experiments are shown in Fig.3.

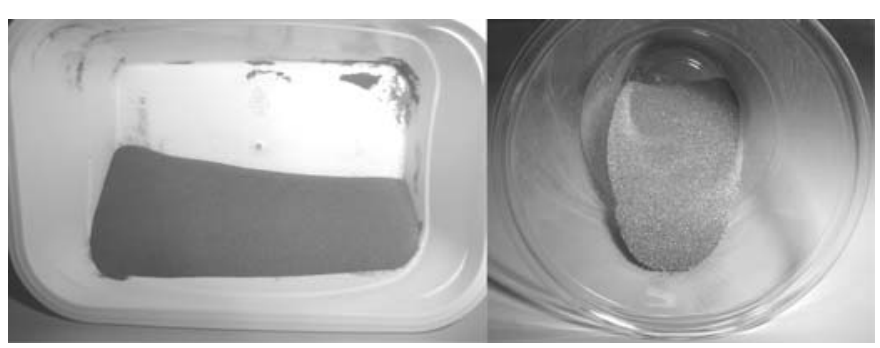

Fig. 3. $100 \mu \mathrm{m}$ and $300 \mu \mathrm{m}$ iron filings.

Carrier fluid must be a lubricant with low viscosity, to get a quite fluid MR suspension. In the proposed MR fluids, commonly available lubricating oil for firearms has been used. Stabilising agents are additives used to improve 
durability and corrosion resistance, and to inhibit the formation of a hard sediment, letting MR fluids to be operational after long inactivity periods with an easy shake. Simplest stabilising agents are greases. In this work, low cost white lithium grease, known in naval activities for its humidity resistance, has been used. An approximately $100 \mathrm{ml}$ of MR fluid recipe uses $150 \mathrm{~g}$ of iron filings, $55 \mathrm{~g}$ of oil and $5 \mathrm{~g}$ of white lithium grease, resulting in a fluid having about $21 \%$ iron by volume [4]. As regards the manufacturing procedure, first, the grease has been dissolved manually into the oil with a stick and a plastic bottle was shaken for five minutes. The suspension has been rested for few hours, to let the grease to complete its dissolution into the oil, and then shaken again for a few minutes. Finally, iron powder has been added, keeping on shaking until the moisture appeared relatively uniform.

\section{Electric and magnetic characterisation}

Relative magnetic permeability has been measured by a homemade solenoid. It was a hollow plastic cylinder, wounded with 520 wire turns, showing a resistance of 11 $\Omega$, and filled with MR fluid that acts as a core material. By applying a sinusoidal voltage input to the circuit, a rather simple voltage-current-frequency measurement yields the inductance value, which can be compared with the one obtained with an air core, to get the fluids' relative magnetic permeability. Measurements on the air core solenoid have revealed an inductance value of $L_{\text {air }}=2,16 \mathrm{mH}$. Measurements with MR fluid cores have been performed in two ways. First, a sinusoidal input with a frequency sweep from $100 \mathrm{~Hz}$ to 2 $\mathrm{kHz}$ was applied. For the MR fluids with $100 \mu \mathrm{m}$ and $300 \mu \mathrm{m}$ filings, the relative permeability were of $\mu_{r}=1.84$ and $\mu_{r}=1.75$, respectively. A $1 \mathrm{kHz}$ sinusoidal input with a current sweep from 0 to $0.1 \mathrm{~A}$ was applied afterwards. In this case, the relative permeability were of $\mu_{r}=1.72$ and $\mu_{r}=1.65$.

In addition, MR fluid's resistivity has been measured with a homemade device, which is essentially a cylindric plastic body of $5 \mathrm{~mm}$ diameter, filled with MR fluid, and with two copper electrodes at both endings. The measurements were performed both without any external magnetic field and by positioning the body vertically inside the solenoid, to consider the worst case in which iron particles form columnar structures along the cylinder. Instrumentation readings have always shown an infinite resistance. Evidently, this is because carrier oil and grease act as insulating materials, and no eddy currents originate in the MR fluid.

\section{Design of the prototype}

In order to analyse rheological properties of the homemade MR fluids, a force feedback system, here used as a viscometer, has been designed and manufactured. The major reason for this step was the need of a device capable of measuring rheological properties of MR fluids as function of an external applied magnetic field. As stated in the introduction, specific instruments are not common, in particular in non-specialised laboratory as electric drives ones.
Also sizing is worth a comment. Actually, the prototype is somewhat bulky, since it is an halfway between a force feedback system and an instrumentation device. Most likely, next research steps will lead to a smaller and lighter one, intended for direct coupling with the steering wheel of the vehicle.

\subsection{Mechanical structure design}

A 2-D view of the proposed MR fluid-based device is shown in Fig.4. MR fluid is contained in an aluminium glass supported by an iron base.

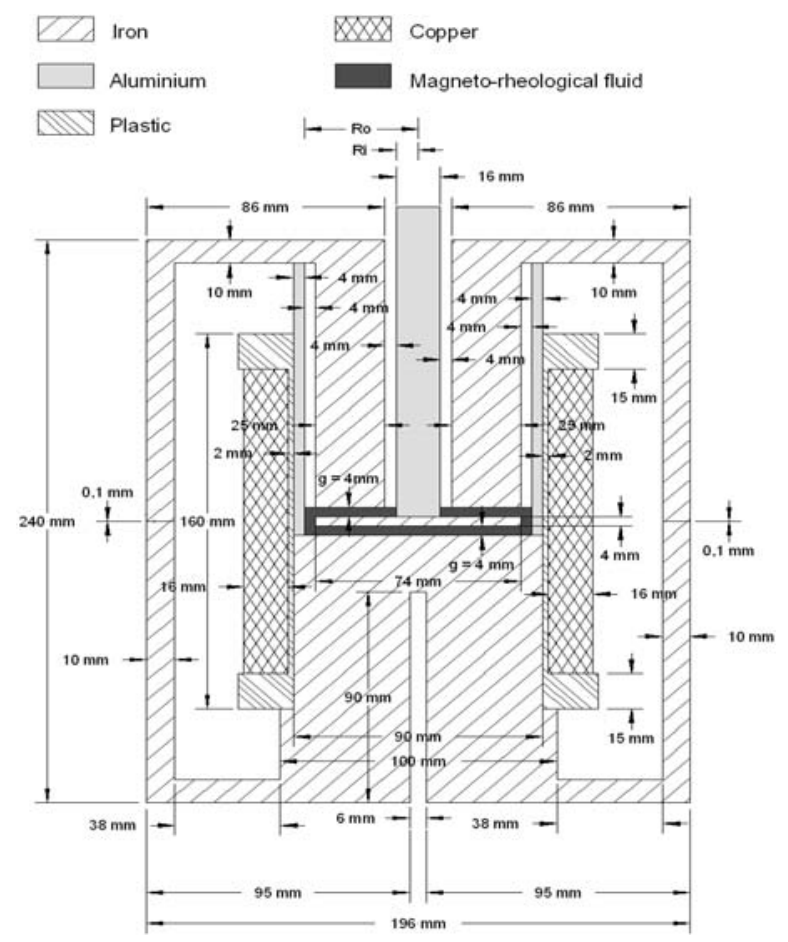

Fig. 4. Mechanical structure of the force feedback system.

Iron surrounds the aluminium glass and the MR fluid forming a magnetic circuit, in which the fluid is the only gap with increased reluctance. A hole in the iron base allocates a thermistor to monitor the internal temperature. Excitation coil features 2000 turns wounded around a plastic reel and inserted the iron base and the aluminium glass, as shown in Fig.5.

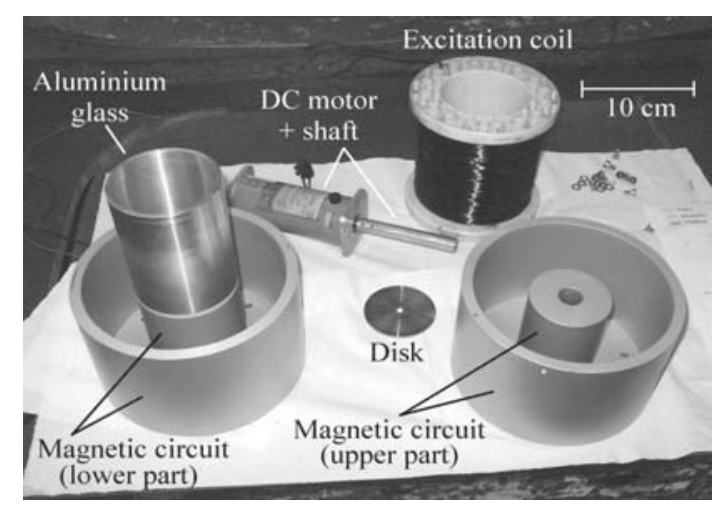

Fig. 5. Separate parts of the manufactured force feedback system. 
The core of the system is an iron disk, dipped into the MR fluid, and linked by an aluminium shaft to a speed-controlled $24 \mathrm{~V}$, 3A DC motor, with a rated torque of $0.4 \mathrm{Nm}$. The actual DC motor torque, proportional to the varying gap fluid viscosity, was acquired as system output data. By using the Equation (2), it has been demonstrated (Appendix A) that the load torque exerted by the MR fluid is equal to:

$$
\tau_{\text {tot }}=\frac{2}{3} \pi T_{c}(H)\left(2 R_{0}^{3}-R_{i}^{3}\right) \operatorname{sgn}(\Omega)+\left(\frac{\pi \eta \Omega}{2 g}\right)\left(2 R_{0}^{4}-R_{i}^{4}\right)
$$

where $\Omega$ is the angular speed of the disk, $g$ is the one-side gap and $\mathrm{R}_{0}, \mathrm{R}_{\mathrm{i}}$ are the internal and outer radii of the disk (Fig.4).

\subsection{Excitation coil design}

The coil has been designed as large as possible in order to obtain a wide magnetic field variation into the device. The wire diameter was $1 \mathrm{~mm}$ and the coil was $130 \mathrm{~mm}$ high, with internal and external diameters of $94 \mathrm{~mm}$ and $126 \mathrm{~mm}$, respectively. From these specifications and by supposing an excitation current of $0.1 \mathrm{~A}$, a mathematical prediction of the flux density of $25 \mathrm{kA} / \mathrm{m}$ was derived. Using for example $\mu_{r}=1,75$, measured for the $300 \mu \mathrm{m}$ MR fluid, the flux density results of $B \approx \mu_{r} \mu_{0} H \approx 0.055 T$. This is confirmed by a finite element analysis of the force feedback system, whose output plot is reported in Fig.6.
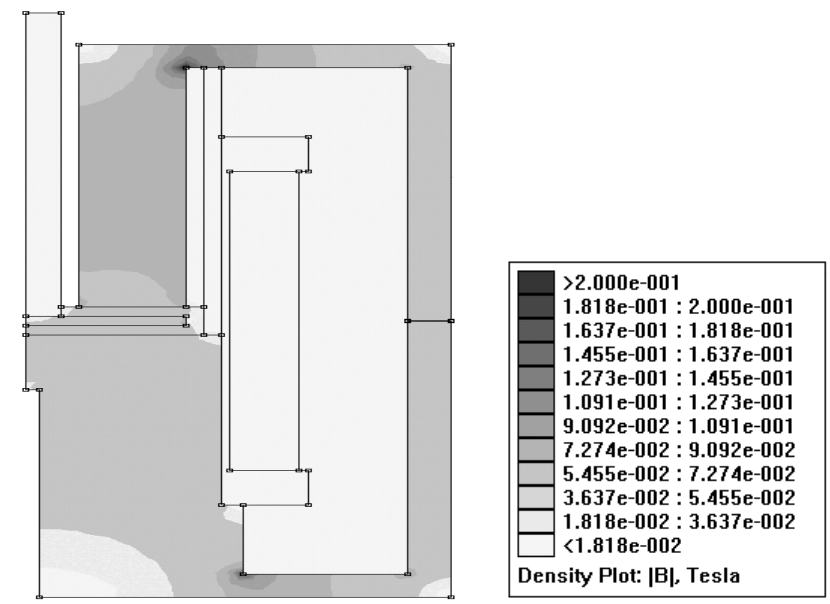

Fig. 6. FEM analysis of the force feedback system structure.

Slightly different results should be attributed to both the presence of the air gap between the aluminium shaft and the iron path, necessary for the shaft movement (low flux density of Fig.6) and to disk end-effects. The coil temperature has been monitored, too. With a 3 A current, a linear increase in temperature from $27^{\circ} \mathrm{C}$ to $41^{\circ} \mathrm{C}$ has been observed, with a gradient of $0.037{ }^{\circ} \mathrm{C} / \mathrm{s} .27 \mathrm{~s} /{ }^{\circ} \mathrm{C}$. Since the plastic reel tolerates a maximum temperature of about $50^{\circ} \mathrm{C}$, above results indicates that forced ventilation was not necessary.

\section{Experimental setup}

Fig.7 shows a view of the complete experimental setup. Since yield stress value $T_{c}(H)$ for $H=0$ is zero, first measurements without current excitation has been made. Equation (3) gives $\eta$ from geometrical parameters and speed measurements.

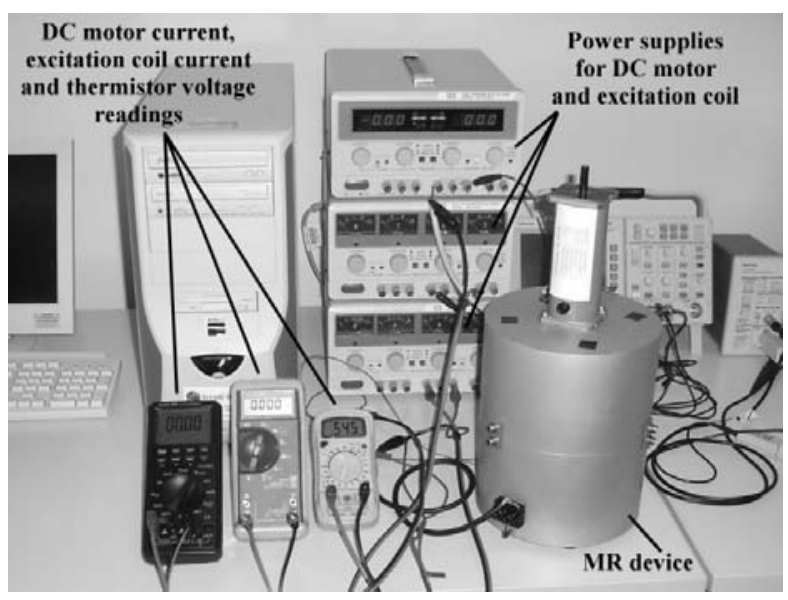

Fig. 7. The complete experimental setup.

For example, the value of $\eta$ for the $100 \mu \mathrm{m}$ MR fluid was $0.508 \mathrm{~Pa}^{*}$ s. Successive measurement sets have been made considering different disk speed and currents in the excitation coil. The load torque exerted by the $100 \mu \mathrm{m}$ MR fluid, as function of the excitation current, is reported in Fig.8, which confirms the quadratic profile of shear stress for low field values mentioned in the literature [8].

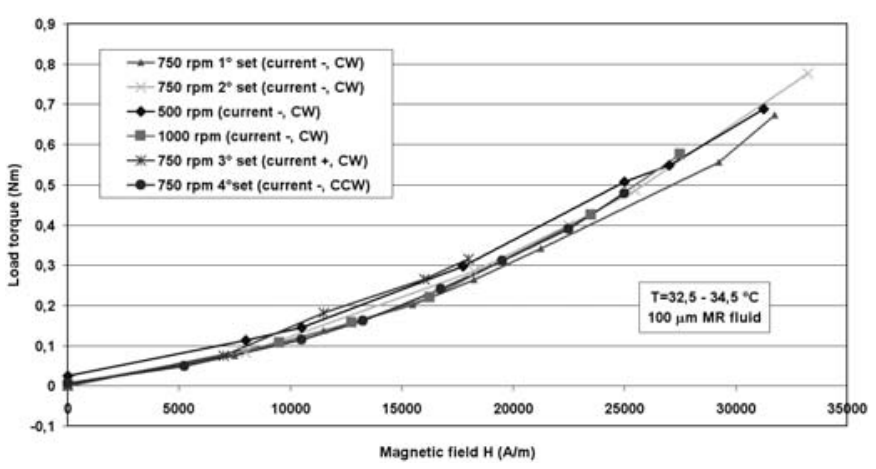

Fig. 8. Motor load torque as function of applied magnetic field.

According to the specification of a major car manufacturer, the force feedback system coupled to the steering wheel has to be able to generate a continuous torque of $6.5 \mathrm{Nm}$. Fig.8 shows that with a magnetic field of $25 \mathrm{kA} / \mathrm{m}$ the developed torque was of about $0.5 \mathrm{Nm}$, limited only by the DC motor ratings. As a design hint for the industrialised device, it has been found that the specified reaction torque can be obtained with a magnetic field of approximately $105 \mathrm{kA} / \mathrm{m}$.

After the steady state characterisation, the dynamic behaviour was investigated, too. The response of the force feedback system to a step of the excitation current was modelled with a simple first-order transfer function:

$$
F_{M R}(s)=\frac{k_{M R}}{1+s \tau_{M R}}
$$

A constant voltage supplied the DC motor, while a stepvoltage was applied to the excitation coil. The identification of the system (4) was obtained by exploiting the knowledge of the electrical and mechanical parameters of both the DC 
motor and the excitation coil. Fig.9 shows both the simulated and the experimental motor load torque, following a $45.5 \mathrm{~mA}$ reference step of the excitation current.

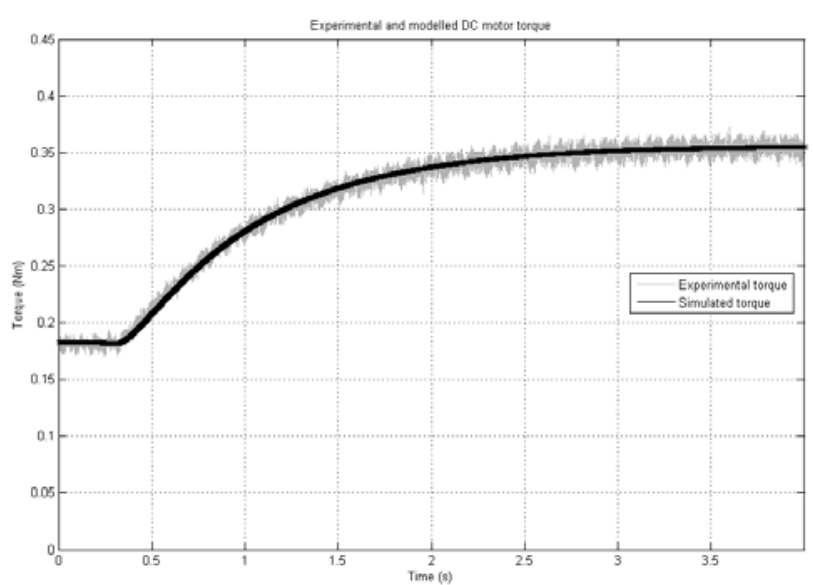

Fig. 9. Force feedback step response to an excitation step variation.

The identified parameters were $\mathrm{k}_{\mathrm{MR}}=4,98$ and $\tau_{\mathrm{MR}}=0,306$. The relatively high value of $\tau_{\mathrm{MR}}$ can be justified by the thickness of iron particles within the fluid $(300 \mu \mathrm{m})$.

The specification on the force feedback given by the car manufacturer indicates a desired bandwidth around $4 \mathrm{~Hz}$. It is believed that with smaller particles, the dynamic behaviour will be quite improved, and the associated time constant will easily meet the specification.

\section{Conclusions}

This paper has presented the design of a force feedback prototype oriented to steer-by-wire applications. To the purpose, two low-cost, homemade MR fluids have been prepared and tested. Accurate steady state and dynamic characterisation of MR fluids has been accomplished as well. As a whole, the paper gives the basic hints for a proper design of a lighter and smaller device, to be mounted under the steering wheel of a common vehicle, acting as a semi-active force feedback device. Future works will involve a deep analysis of MR fluids' dynamic properties, and the design and manufacturing of the final actuator.

\section{Acknowledgements}

The authors would like to thank Metalpolveri s.r.l (Brescia, Italy), CTS (Conegliano, Italy) and Hoganas Italia s.r.l. (Genova, Italy) for their special collaboration in the experimental phase of this work.

\section{References}

[1] J. An, D-S. Kwon, "Modelling of a Magnetorheological Actuator Including Magnetic Hysteresis", Journal of Intelligent Material Systems and Structures, volume 14, pp. 541-550, (2003).

[2] A.Benedetti, N.Bianchi, S.Bolognani, M.Dai Prè, P.G.Molari, M.Tomasini, L.Tubiana, M.Zigliotto, "PM
Motor Drives for Steer-by-Wire Applications", Conf. Rec. of IEEE-IAS, pp. 2857-2864, Hong Kong, (2005).

[3] S.Bolognani, M.Tomasini, M.Zigliotto, "Control Design of a Steer-by-Wire System with High Performance PM Motor Drives", Proc. of IEEE-PESC, pp.1839-1844, Brasil, (2005).

[4] J. D. Carlson, "Do-It-Yourself Magnetorheological Fluid”, www.lord.com.

[5] J. D. Carlson, D. M. Catanzarite, K. A. St. Clair, "Commercial Magnetorheological Fluids Devices", www.lord.com.

[6] S.K.Cobb, Y.M.Shkel, "Electromagnet for magnetorheological testing of solid and liquid magnetic suspensions", Proc. of 74th Ann. Meet. of the Society of Rheology, USA, (2002).

[7] S. Genç, "Synthesis and Properties of Magnetorheological (MR) Fluids”, Ph.D. Thesis, University of Pittsburgh, (2002).

[8] M. R. Jolly, J. W. Bender, J. D. Carlson, "Properties and Applications of Commercial Magnetorheological Fluids", www.lord.com.

[9] Lord Corp., "Tactile Feedback Devices Product Bulletin”, www.lord.com, (2005).

\section{Appendix - Proof of Equation (3)}

The total load torque exerted by the MR fluid can be calculated from Equation (2), referred to the axisymmetric structure of Fig.4. By supposing that the MR fluid layer in contact with the iron disk's upper plate rotates with an angular velocity $\Omega$, and the MR fluid layer contacting the iron surface remains still, the shear rate is a function of the radius $r$ alone [1]:

$$
\dot{\gamma}(r)=\frac{r \Omega}{g}
$$

$r \Omega$ represents the tangential velocity of a generic point located at circumference with radius $r$. By substituting this expression in Equation (2), it follows:

$$
T=T_{c}(H) \operatorname{sgn}(\dot{\gamma})+\eta \frac{r \Omega}{g}
$$

The shear stress at radius $r$ can be expressed as a torque, if one considers that a differential donut element contributes with a differential torque of:

$$
d T=T(r) \cdot 2 \pi r^{2} d r
$$

Integrating over $d T$ from $R_{i}$ to $R_{o}$ leads to:

$$
\tau_{u p}=\frac{2}{3} \pi T_{c}(H)\left(R_{0}^{3}-R_{i}^{3}\right) \operatorname{sgn}(\Omega)+\frac{\eta \pi \Omega}{2 g}\left(R_{0}^{4}-R_{i}^{4}\right)
$$

Disk's lower side torque contribute is easier to determine, since in this case internal radius $R_{i}=0$. The integration over $d T$ with $R_{i}=0$ gives:

$$
\tau_{\text {low }}=\frac{2}{3} \pi T_{c}(H) R_{0}^{3} \operatorname{sgn}(\Omega)+\frac{\eta \pi \Omega}{2 g} R_{0}^{4}
$$

The total load torque exerted by the MR brake-viscometer is the sum of $\tau_{u p}$ and $\tau_{\text {low }}$, that is, Equation (3). 